\title{
COMUNICAÇÃO CIENTÍFICA E ATENÇÃO ONLINE: EM BUSCA DE COLÉGIOS VIRTUAIS QUE SUSTENTAM MÉTRICAS ALTERNATIVAS
}

\section{COMUNICACIÓN CIENTÍFICA Y ATENCIÓN EN LÍNEA: EN BÚSQUEDA DE COLEGIOS VIRTUALES QUE APOYAN LAS ALTMÉTRICAS}

\author{
Ronaldo Ferreira de Araújo* \\ Ariadne Chloe Mary Furnival ${ }^{* *}$
}

\begin{abstract}
RESUMO
Introdução: As trocas informacionais em torno da atividade de comunicação científica têm se tornado cada vez mais dinâmicas no ciberespaço, modificando a prática dos cientistas e ampliando o alcance dos resultados de pesquisa que atinge uma atenção online cada vez mais expressiva entre grupos distintos.

Objetivo: Analisar se os dados altmétricos de atenção online apontam a existência de "colégios virtuais" - compostos por pessoas de dentro e de fora da comunidade científica - sobre o tema científico sendo compartilhado e comentado nas mídias sociais.
\end{abstract}

Metodologia: Estudo piloto, caracterizado como exploratório, tendo como foco de análise a repercussão do artigo de acesso aberto com o maior indicador altmétrico $(n=2.728)$ para o ano de 2015, segundo divulgado pela Altmetric.com em seu ranking "Top 100". A qualificação da atenção online é descrita pelas mídias mais expressivas (Twitter e Facebook) em uma amostra randômica $(n=10 \%)$ para a qual se procedeu a análise de conteúdo e para caracterização do perfil dos grupos sociais que interagiram e a categorização dos tipos de conteúdos de interação.

Resultado: A forma mais comum de disseminação observada foi 0 de compartilhamento e repasse para o Twitter (62\%) e Facebook (45\%), seguido das categorias "conclusivo" (16\% e 25\%) e argumentativo (15\% e 9,6\%). O perfil dos usuários responsáveis pela circulação do artigo indica se tratar de especialistas da área da saúde.

Conclusão: Embora a altmetria proponha uma avaliação de impacto mais social e democrático da pesquisa científica os colégios virtuais caracterizados indicam um público especializado e o debate e circulação da informação científica sobre o artigo analisado se concentra na comunidade científica.

Palavras-chave: Comunicação científica. Altmetria. Atenção online. Colégios Virtuais.

* Doutor em Ciência da Informação (UFMG). Professor do Curso de Biblioteconomia, Universidade Federal de Alagoas, UFAL. E-mail: ronaldo@gmail.com.

** Doutora em Política Científica e Tecnológica (UNICAMP). Professora do Programa de PósGraduação em Ciência da Informação, Universidade Federal de São Carlos, UFSCar. E-mail: chloe@ufscar.br. 


\section{INTRODUÇÃO}

As trocas informacionais que ocorrem em torno das atividades de comunicação científica têm se tornado cada vez mais dinâmicas no ciberespaço, modificando a prática dos cientistas e ampliando o alcance dos resultados parciais e finais de pesquisa que atingem uma atenção online cada vez mais expressiva entre grupos distintos.

Nesse contexto, os ambientes digitais, as redes e mídias sociais como dispositivos informacionais possuem dupla função e atuam tanto como filtros para obtenção de informação relevante ou fontes para estabelecimento de contatos entre pesquisadores, cientistas e público em geral.

De acordo com Moreira (2005) a comunicação científica e suas novas ferramentas e possibilidades interativas ou de interação presentes na web ressurgem como campo complexo de investigação, e são necessários novos estudos com o fim de verificar onde e como se dão as novas confluências. Para o autor as inter-relações com os grupos sociais e as comunidades de prática, e as formas de interação entre os cientistas ainda carecem de estudos mais sistemáticos.

Uma vez que se dedica a compreensão dos indicadores de engajamento, debate e outros tipos de interação em torna da informação científica na web social, a altmetria é um fértil campo para preencher essa lacuna de estudos. As métricas alternativas podem contribuir na identificação de assuntos ou temas populares nas mídias sociais; oferecer uma visão panorâmica do que tem recebido atenção online; e identificar grupos de interesse ou comunidades de atenção.

A presente pesquisa objetiva analisar se os dados altmétricos de atenção online apontam a existência grupos ou "colégios virtuais" - compostos por pessoas de dentro e de fora da comunidade científica - sobre o tema científico sendo comentado e compartilhado nas mídias sociais. Para tanto discorre sobre a comunicação científica e internet ressaltando aspectos da visibilidade e atenção online; reflete brevemente sobre a altmetria como possibilidade de identificação de colégios virtuais que sustentam seus dados. 
Por fim apresenta um estudo piloto que teve como foco rastrear repercussão de um artigo e qualificar sua atenção online recebida.

\section{COMUNICAÇÃO CIENTÍFICA, INTERNET E ATENÇÃO ONLINE}

A atividade científica é realizada em meio a inúmeras trocas e interações entre atores sociais, sendo a informação um de seus elementos principais. Autores como Le Coadic (1996, p.27) a considera tão vital que chega a dizer que "a informação é o sangue da ciência". Assim sendo, sem informação, "a ciência não pode se desenvolver e viver. Sem informação a pesquisa seria inútil e não existiria o conhecimento. Fluido precioso, continuamente produzido e renovado, a informação só interessa se circula, e, sobretudo, se circula livremente" (LE COADIC, 1996, p.27).

É no campo da comunicação científica que a informação concretiza seu papel fundamental de publicização e divulgação do conhecimento. Tal campo pode ser considerado como área de estudo das "atividades informacionais que ocorrem entre os produtores da informação científica, desde o momento em que eles iniciam suas pesquisas até a publicação de seus resultados e sua aceitação e integração a um corpo de conhecimento científico" (GARVEY, 1979, p. 10).

Para Targino (2000) a comunicação científica é indispensável à atividade científica, pois permite somar os esforços individuais dos membros das comunidades científicas.

Eles trocam continuamente informações com seus pares, emitindo-as para seus sucessores e/ou adquirindo-as de seus predecessores. É a comunicação científica que favorece ao produto (produção científica) e aos produtores (pesquisadores) a necessária visibilidade e possível credibilidade no meio social em que produto e produtores se inserem (TARGINO, 2000, p.10).

Com o desenvolvimento das tecnologias de informação e comunicação, em especial, dos computadores e das redes eletrônicas, as formas de comunicação disponíveis à comunidade científica vêm se modificando, 
ampliando e diversificando, tornando-se cada vez mais eficientes, rápidas e abrangentes, vencendo barreiras geográficas, hierárquicas e financeiras (MUELLER, 2000).

Pode-se acrescentar aos avanços dessas tecnologias o surgimento e amplo uso da internet, que de acordo com Silva, Menezes e Bissani (2002), como meio de comunicação, caracteriza-se pelo seu poder globalizador e pela instantaneidade com que os documentos podem ser produzidos, divulgados, atualizados e acessados. Segundo as autoras, a web merece especial destaque devido sua capacidade de

hipermídia (hipertexto e multimídia) que facilita a procura e a divulgação de informação. Possibilita, além de acesso as fontes inesgotáveis de informação, comunicação a baixo custo. Permite a interconexão de usuários para os mais variados fins e tem contribuído para democratizar e socializar o acesso à informação, eliminando barreiras relacionadas à distância, ao tempo, aos problemas políticos e sociais, etc (SILVA; MENEZES; BISSANI, 2002, p.2).

Para Neubert e Rodrigues (2012, p.143) da mesma forma que possibilitou novas formas de divulgação da produção científica, a internet "modificou a maneira com que os pesquisadores se comunicam, trocam experiências e informações a respeito de suas pesquisas".

Ao comparar o fluxo da comunicação científica tradicional e da comunicação científica eletrônica, Castro (2006) afirma que no segundo caso, com o advento da internet e o desenvolvimento de novas tecnologias, alteraram-se as relações sociais e o fluxo da comunicação científica é reestruturado. Nesse ponto, ressalta-se a importância da internet e, em especial, de que "seu impacto na comunicação científica pode ser entendida pela análise de seu desenvolvimento e de algumas de suas características, tais como a interatividade, o compartilhamento e a temporalidade" (OLIVEIRA; NORONHA, 2005, p.79).

Tais mudanças ocorrem nas várias etapas do fluxo da informação científica e se estendem aos meios (formais e informais) nos quais ela é comunicada. A comunicação informal sempre foi vista como efêmera, geralmente expressada de forma oral, de alcance limitado e de difícil 
compreensão e análise por não estarem disponíveis de forma aberta (MEADOWS, 1999).

De acordo com Araújo e Freire (1996, p.12) os canais informais de comunicação "são altamente eficientes na perspectiva da interação direta entre a fonte e o usuário da informação, diminuindo as barreiras na transferência da informação por utilizarem a informação interpessoal".

\begin{abstract}
E quando se pensa que os canais informais são fundamentais para se trabalhar em níveis de maior valor agregado à informação, porque são úteis na tomada de decisão e possibilitam a criação de estratégias a partir de informações ainda não publicadas, pode-se ter a ideia do valor comunicacional da rede (ARAÚJO; FREIRE, 1996, p.53).
\end{abstract}

Se a internet desempenhava um papel facilitador na comunicação informal entre pesquisadores nas diversas áreas de Ciência \& Tecnologia (ARAÚJO; FREIRE, 1996), agora com a evolução da web e os recursos da web 2.0 esse tipo de comunicação desperta a atenção online, amplia o alcance de pesquisadores a outros interessados, leitores e debatedores dentro e fora da comunidade científica. Estudos como o de Fausto et. al (2012) confirmam os blogs científicos, por exemplo, como ricos espaços para o debate que sintetizam informações relevantes de pesquisa e promovem uma compreensão mais profunda dos assuntos que discutem.

Outro aspecto é que se os canais informais já apresentavam a vantagem de permitir aos cientistas descobrirem rapidamente se estão falando dos mesmos problemas e se os temas abordados são de mútuo interesse (GARVEY; GRIFFITH, 1967) com a circulação de suas pesquisas na websocial eles têm uma noção, imediata à publicação, quanto à atenção online que recebem, bem como a aceitação do público.

A atenção online tem sido compreendida como aspectos de audiência (alcance) e interações que conteúdos recebem nas mídias sociais (ARAUJO, 2015). Assim, pesquisadores e outros interessados conseguem identificar dentre as pesquisas atuais, quais temas e assuntos são mais populares e atraem mais interlocutores. 
Nesse contexto, e dada a visibilidade e atenção online que a informação cientifica alcança, autores como Moreira (2005, p.58) utiliza a expressão "colégio virtual" como analogia a "colégio invisível", segundo o autor "o colégio virtual atua como rede de comunicação e intercâmbio, como fórum de educação e de socialização dos novos cientistas".

Com o aumento de ferramentas capazes de acompanhar a audiência e a atenção online da circulação da informação científica tais dados são passíveis de monitoramento e podem sinalizar a presença de colégios virtuais. A altmetria, campo que analisa o quanto um artigo é compartilhado, debatido ou avaliado e por quem ele recebe tais interações na web social oferece condições ideiais para a compreensão dos grupos sociais que sustentam suas métricas.

\section{ALTMETRIA E A POSSÍVEL IDENTIFICAÇÃO DE COLÉGIOS VIRTUAIS}

Segundo a "Altmetrics Manifesto", a altmetria é "a criação e estudo de novas métricas baseadas na Web Social para análise e para estudos informacionais" (PRIEM et al., 2010). Trata-se de métricas sociais que incorporam as atividades de bookmarking e disseminação, cobertura em blogs e nos sites da mídia, discussão, apreciações e avaliações (ratings). Para tanto, procura medir a disseminação e compartilhamento de informações científicas de várias naturezas por meio das ferramentas da web social.

A altmetria pode complementar os estudos métricos tradicionais, permitindo avaliar o impacto de uma pesquisa científica para além do número de citações que recebe, e até mesmo para além do âmbito das comunidades científicas.

O surgimento da altmetria tem sido pautado pelo contexto digital e densamente enredado em que os pesquisadores científicos inevitavelmente trabalham quando se fala de Digital Scholarship, que significa o uso de evidências digitais, métodos de pesquisa, publicação e preservação para atingir as metas acadêmicas e de investigação, podendo abranger tanto a comunicação científica usando mídia digital quanto a própria pesquisa em 
mídia digital, que combinados com acesso aberto oferece um modelo mais acessível e aberto para a atividade científica (RUMSEY, 2011; HORNBY; LESLIE, 2013).

A visibilidade e atenção online tem sido considerada um imperativo dos últimos tempos e mesmo se, por algum motivo um pesquisador não estiver inserido no ambiente online do nexo digital que caracteriza a vida do século $\mathrm{XXI}$, suas pesquisas estão, seja por meio das revistas eletrônicas, ou das bases de dados e repositórios, ou websites pessoais ou de compartilhamento de arquivos (como Figshare, Slideshare etc.). E os usuários já estão "citando" essas pesquisas nos grupos coletivos em que participam.

A altmetria trata de uma nova forma de perceber o "uso" e citação da informação científica, que antes da citação convencional em outra publicação científica, ganha visibilidade nestas menções, curtidas e compartilhamentos nas redes e mídias sociais.

Os dados altmétricos indicam a atenção que os artigos (e outras formas de informações científicas) recebem logo após a publicação e podem ser indícios de citações futuras. São métricas que consistem na repercussão que artigos científicos alcançam em portais de notícias, blogs, mídias sociais como Facebook, Twitter, Google+ e serviços de referência como Mendeley e CiteUlike. De acordo com Cave (2012, p.349-50) e Tanabaum (2013, p.5), as altmétricas são usualmente categorizadas da seguinte forma:

Uso: visualizações em HTML, PDF/SML, downloads. Quantas vezes um artigo tem sido visualizado no site da editora? Quantas vezes tem sido acessado ou baixado o texto completo? Quantas vezes tem sido acessado os dados suplementais ao artigo? Estas cifras dão um senso concreto e tangível sobre o quanto um artigo está de fato sendo lido.

Captura: bookmarks, favoritos, leitores, grupos. Com quão frequência um artigo foi marcado como favorito (bookmarked) no CiteULike, Evernote etc.? Quão frequentemente tem sido compartilhado no Mendeley? Estes e outros "sistemas de recomendação" provem um insight sobre qual pesquisa os pesquisadores querem realçar entre seus pares.

Menções: postagens em blogs, noticiarias, artigos de wikipedia, comentários, criticas. Que é a discussão centrada em torno do artigo? Quantas vezes foi blogado? Quantas notícias tem sido escritas sobre ele? Qual é a frequência de suas menções na Wikipedia? Quantos comentários gerou na website da editora e em 
outros sítios? Esta dimensão procura quantificar até que ponto um artigo tem penetrado além da audiência estreitamente acadêmica e se tem gerado uma conversa mais ampla.

Mídias sociais: atividade dos usuários de Facebook, Twitter, Reddit, Google+ etc. Quantas curtidas no Facebook tem o artigo? Quantas vezes foi compartilhado no Linkedln e outras redes sociais? Quantos tweets gerou o artigo? Estas medidas podem contribuir ao senso de atualidade, imediação do artigo.

Os questionamentos levantados dentro de cada uma das categorias realça o aspecto mais "imediato" e transitório das altmétricas, devido à natureza muito dinâmica e "do momento" das mídias sociais nas quais usualmente circulam. Mas, outro aspecto das altmétricas que podemos enfatizar é que elas são mais dialógicas no seu impacto quando comparadas àquelas puramente bibliométricas, já que usualmente contém comentários com o criador do item sendo lido e comentado, podendo, assim, apontar para o tipo de apropriação e uso do item sob certo escrutínio.

A rede social acadêmica ResearchGate $<$ www.researchgate.net/ $>$, por exemplo, que já registrou mais de 8 milhões de membros (BENGSCH, 2015) permite ao usuário, 'seguir' (follow) publicações de seu interesse e avaliá-las ou mesmo realizar o upload de suas próprias publicações e acompanhar a atenção que as mesmas recebem em termos de leituras, podendo inclusive solicitar aos leitores um feedback ou comentário sobre a publicação.

O Mendeley < https://www.mendeley.com/> é outro exemplo que pode ser mencionado. O dispositivo tem sido particularmente bem-sucedido entre os gerenciadores de referências online, com indicativo de mais de 1,6 milhões de usuários e mais de 161 milhões de documentos, os quais quando salvos em sua biblioteca é considerado um indicador de leitura (PRIEM; PIWOWAR; HEMMINGER, 2012). Quando se analise itens únicos é possível visualizar o perfil dos leitores quanto à área de atuação, titulação acadêmica e região geográfica.

Para além das leituras e pensando em outros tipos de interações, o Facebook foi objeto de estudo de atenção online expressa por meio de curtidas, comentários e compartilhamentos de artigos de periódicos da Ciência da Informação na pesquisa de Araújo e Murakami (2016). Os autores 
consideraram as interações com conteúdos de artigos científicos no Facebook ricas fontes de dados altmétricos, podendo ser benéficas como uma métrica agregada por fornecerem uma visão adicional sobre o desempenho científico dos artigos quando curtidos, compartilhados ou comentados.

Assim, é possível que os dados altmétricos revelem o acesso e leitura, bem como outras interações em torno da informação científica por pessoas externas à comunidade acadêmica, e também de pesquisadores de outros campos. Em ambos os casos - dentro e fora da comunidade científica - 0 pressuposto é o de que os grupos que lêem, comentam e compartilham podem ser considerados colégios virtuais.

Com tais ações de informação, a comunicação informal, conhecida e expressa nos denominados colégios invisíveis, que por sua vez, que sempre foi comum entre os cientistas, se reconfiguram nesses ambientes digitais como "colégios virtuais" e passam a ser ainda mais interativos e democráticos, facilitando a avaliação da produção científica (GURGEL; MATOS, 2006) e o consequente reconhecimento científico e social dos cientistas.

Os colégios virtuais podem ser mapeados a partir das diferentes formas de interação e circulação da informação científica nas mais variadas mídias sociais e dispositivos informacionais. Ao serem identificados os colégios virtuais vão indicar verdadeiras "comunidades de atenção" (HAUSTEIN; BOWMAN; COSTAS, 2015) sobre o tema em questão, e que como compartilham essa informação pode revelar traços das ações de informação desse grupo.

\section{METODOLOGIA}

Em relação aos procedimentos metodológicos adotados, nosso estudo piloto pode ser caracterizado como exploratório, centrando-se no levantamento, sistematização e análise de dados altmétricos tendo como foco de análise a repercussão do artigo de acesso aberto com o maior indicador altmétrico para o ano de 2015, segundo divulgado pela Altmetric.com em seu ranking "Top 100 ARTICLES - 2015" < https://www.altmetric.com/top100/2015/\#explore >. Optamos pelo critério de artigo de acesso aberto por entender que tal 
Ronaldo Ferreira de Araújo; Ariadne Chloe Mary Furnival

Comunicação científica e atenção online: em busca de colégios virtuais que sustentam métricas alternativas

disponibilidade possa implicar que 0 artigo tivesse um alcance além da comunidade científica, que frequentemente se dispõe de acesso subsidiado às revistas científicas de assinatura, não disponíveis em acesso aberto. A Figura 1 mostra os cinco primeiros colocados no ranking.

Figura 1. Artigos com dados altmétricos mais expressivos

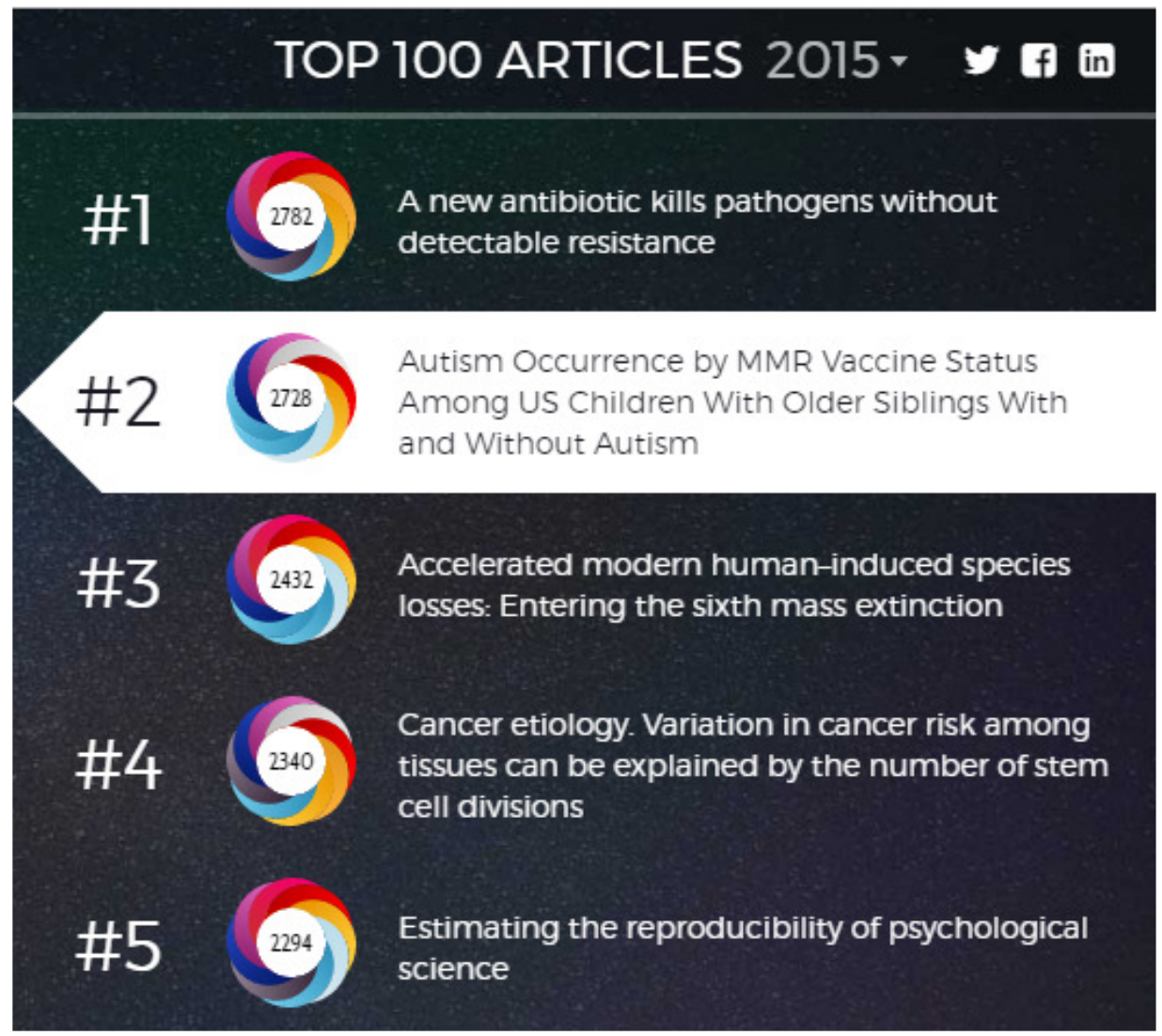

Fonte: Altmetric.com "Top100 ARTICLES - 2015".

O artigo da primeira posição, "A new antibiotic kills pathogens without detectable resistance", publicado na Nature, é de acesso restrito. O segundo colocado, "Autism Occurrence by MMR Vaccine Status Among US Children With Older Siblings With and Without Autism", publicado na The Journal of the American Medical Association - JAMA, está disponível em acesso aberto, e conforme critério estabelecido, seguimos com uma análise quali-quantitativa do conteúdo das conversações e interatividade sobre este artigo, visando traçar os entornos das comunidades de atenção, por meio de amostragem randômica para as menções. 
Primeiramente descreve-se a atenção online recebida pelo artigo nas diversas fontes e mídias, em seguida caracteriza-se o público que interage com o artigo por meio da descrição do perfil e distribuição geográfica. Para a qualificação da atenção online dedica-se a análise das mídias mais expressivas (Twitter e Facebook) em uma amostra randômica ( $n=10 \%)$ para a qual se procedeu a categorização dos tipos de interação.

Trabalhamos com seis categorias que são listadas e explicadas no Quadro 1. As categorias foram elaboradas tendo por inspiração o trabalho de Nelhans e Lorentzen (2015) que analisaram quais artigos científicos são mencionados no Twitter e como são mencionados. Utilizamos o procedimento qualitativo de análise de conteúdo, adequada para analisar o contexto de mensagens textuais, sendo um método flexível que pode ser aplicado a muitos problemas de pesquisa na Ciência da Informação, sozinho ou em conjunção com outros métodos (WHITE; MARSH, 2006).

Quadro 1 - Categorias analíticas geradas para o conteúdo das menções

\begin{tabular}{|l|l|}
\hline \multicolumn{1}{|c|}{ Categoria Analítica } & \multicolumn{1}{c|}{ Descrição do conteúdo } \\
\hline $\begin{array}{l}\text { Compartilhamento } \\
\text { (repasse) }\end{array}$ & $\begin{array}{l}\text { Cópia do URL do artigo com uma breve síntese do } \\
\text { conteúdo semelhante ao título do artigo. }\end{array}$ \\
\hline Explicativo & Procuram detalhar mais os resultados do estudo. \\
\hline Argumentativo & $\begin{array}{l}\text { Aferere credibilidade científica do estudo; } \\
\text { Qualifica o estudo como incontestável (devido ao } \\
\text { método, tamanho da amostra, etc); } \\
\text { Se utiliza do estudo para refutar outras teorias (ex. a } \\
\text { relação entre a vacina tríplice e o autismo). }\end{array}$ \\
\hline Exortativo & $\begin{array}{l}\text { Argumentando/discutindo um ponto; } \\
\text { Energicamente a favor da abordagem do estudo; } \\
\text { Lista este estudo entre outros semelhantes. }\end{array}$ \\
\hline Problematizante & $\begin{array}{l}\text { Se utiliza do estudo para sensibilizar práticas e ações } \\
\text { (ex. mensagem de saúde pública a favor da } \\
\text { vacinação). }\end{array}$ \\
\hline $\begin{array}{l}\text { Critica e/ou desqualifica o estudo; } \\
\text { Critica o método do estudo (ex. o estudo se baseia em } \\
\text { dados enviesados); } \\
\text { Problematiza a abordagem (ex. é um estudo } \\
\text { epidemiológico e estatístico e não clínico-qualitativo). }\end{array}$ \\
\hline
\end{tabular}

Fonte: Adaptado de Nelhans e Lorentzen (2015). 
O aporte da análise de conteúdo contribui na construção de inferências replicáveis e válidas de textos (ou outra matéria significativa) aos contextos do seu uso, que permitem aos pesquisadores se moverem do texto às respostas das questões de pesquisa. A análise implica primeiramente na seleção das unidades de amostragem (sampling units) que identificam a população, e que basicamente são os "contêineres documentários", o veículo "natural" das unidades de análise (WHITE; MARSH, 2006).

As unidades de análise são selecionadas para a análise foram os tweets no Twitter e posts no Facebook. Optamos pela análise de conteúdo predominantemente qualitativa, no sentido que é indutiva, sendo que serão as questões da pesquisa que guiam a coleta e análise dos dados, mas novas questões e temáticas podem surgir a partir da leitura cuidadosa e iterativa dos dados. Na prática, isto significa que os esquemas de codificação dos dados analisados são usualmente desenvolvidos durante o processo de das leituras atentas e iterativas das unidades de análise para identificar conceitos e padrões (patterns) significativos (WHITE; MARSH, 2006).

\section{RESULTADOS}

A pontuação Altmetric é uma medida geral da atenção que um artigo ou conjunto de dados tem recebido online, e deve refletir (i) a visibilidade quantidade de atenção recebida - em geral, quanto mais pessoas visualizam ou falam sobre um artigo maior sua pontuação; (ii) influência e engajamento - a qualidade da atenção, local postado e reputação de quem posta (ALTMETRIC, 2014). O valor dos dados altmétricos para o artigo analisado é de 2.728 e sua distribuição pode ser vista na Tabela 1. 
Ronaldo Ferreira de Araújo; Ariadne Chloe Mary Furnival

Comunicação científica e atenção online: em busca de colégios virtuais que sustentam métricas alternativas

Tabela 1 - Distribuição das menções nas mídias sociais do artigo "Autism Occurrence by MMR Vaccine Status Among US Children With Older Siblings With and Without Autism"

\begin{tabular}{l|c}
\hline Canal nas mídias sociais & Dados altmétricos \\
\hline Sites de notícias & 57 \\
\hline Blogs & 30 \\
\hline Twitter & 2.596 \\
\hline Facebook & 39 \\
\hline Google+ & 11 \\
\hline Reddit & 166 \\
\hline Leitores no Mendeley & \\
\hline
\end{tabular}

Fonte: Altmetric.com https://www.altmetric.com/details/3927843

Em termos de atenção online alcançada pelo artigo em valores altmétricos, o Twitter é a mídia mais expressiva (2.596), seguida do Facebook (275). A predominância destas duas redes é recorrente entre os levantamentos dos dados altmétricos (ALPERIN, 2014; ARAÚJO, 2015), uma tendência que, acreditamos, reflete a típica atividade mundial online em 60 segundos, que, para 2015, consistiu em 3,3 milhões de posts feitos no Facebook e 422.340,00 tweets criados no Twitter (ALLEN, 2016).

A caracterização do perfil dos usuários responsáveis pela atenção online do artigo foi prejudicada uma vez que nem todos informam na descrição do perfil sua formação, atuação ou vínculo institucional. De uma forma geral, dada a maioria, em torno de $70 \%$ indica ausência dessas informações. Médicos e outros profissionais da saúde atingem $14 \%$, seguidos por cientistas $12 \%$ e comunicadores da ciência (jornalistas, blogueiros, etc), com 3\%. Os países mais expressivos foram os Estados Unidos (29\%), o Reino Unido (164) e o Canada (6\%).

Por fim, submeteu-se uma amostra randômica de 300 tweets e 31 postagens no Facebook sobre este artigo, que representa aproximadamente $10 \%$ do total número de menções do artigo nestas duas redes sociais, às categorias analíticas sob as quais foi possível agregar o conteúdo das 
Ronaldo Ferreira de Araújo; Ariadne Chloe Mary Furnival

Comunicação científica e atenção online: em busca de colégios virtuais que sustentam métricas alternativas

menções feitas sobre 0 artigo. A Tabela 2 apresenta 0 quantitativo e porcentagem da distribuição das menções por categorias para o Twitter e Facebook e exemplifica (por serem mensagens mais curtas) na última coluna exemplos de tweets enquadrados em cada categoria.

Tabela 2 - Categorias analíticas das menções

\begin{tabular}{|c|c|c|c|}
\hline $\begin{array}{l}\text { Categoria } \\
\text { Analítica }\end{array}$ & Twitter & Facebook & Exemplos (tweets) \\
\hline $\begin{array}{l}\text { Compartihame- } \\
\text { nto (repasse) }\end{array}$ & $\begin{array}{c}186 \\
(62 \%)\end{array}$ & $\begin{array}{c}14 \\
(45 \%)\end{array}$ & $\begin{array}{l}\text { RT @carlsmythe: Absence of link between MMR and } \\
\text { autism https://t.co/6zR6yWKLne } \\
\text { @cgarrett101 JAMA: No increased autism risk from } \\
\text { MMR, even with autistic siblings. } \\
\text { https://t.co/VODYqVZoyi }\end{array}$ \\
\hline Explicativo & $\begin{array}{c}17 \\
(6 \%)\end{array}$ & $\begin{array}{c}2 \\
(6,5 \%)\end{array}$ & $\begin{array}{l}\text { @Charbrevolution No MMR-Autism Link in Large } \\
\text { Study of Vaccinated vs. Unvaccinated Kids } \\
\text { https://t.co/88VIrn296K } \\
\text { @DavidPenington2 Unvaxed sibling of autistic kids as } \\
\text { likely to be autistic as vaxed https://t.co/BGIDQ5OtJr } \\
\text { @tepatiketl RT @tweetmedico: NO hay asociación } \\
\text { entre la vacuna vs el sarampión y autismo inclusive en } \\
\text { niños con } 81 \text { ntecedents de autismo: Estudio http:/... }\end{array}$ \\
\hline Conclusivo & $\begin{array}{c}47 \\
(16 \%)\end{array}$ & $\begin{array}{c}8 \\
(25 \%)\end{array}$ & $\begin{array}{l}\text { @Charbrevolution Did you even read the massive } \\
\text { study of } 90,000+\text { children? https://t.co/88VIrmKyfc } \\
\text { @belligerentMS you can't get any more certain that } \\
\text { this. } 95,000 \text { pt cohort study https://t.co/xfc0ALodvX } \\
\text { @FreelnTX read this one. It is the largest study done } \\
\text { on the topic. } \\
\text { RT @EstebanOrtizMD: Vacuna MMR (triple viral) no } \\
\text { tiene relación con autismo ! MÁS QUE Comprobado } \\
\text { (NUEVAMENTE) }\end{array}$ \\
\hline Argumentativo & $\begin{array}{c}45 \\
(15 \%)\end{array}$ & $\begin{array}{c}3 \\
(9,6 \%)\end{array}$ & $\begin{array}{l}\text { RT @TelaAntkowiak Current evidence disagrees with } \\
\text { that assumption https://t.co.... } \\
\text { @nyalpurcell @stejwill @drpaulmorgan A link to the } \\
\text { study, so you can appraise it and share your criticism } \\
\text { with us: https://t.co/pmaNsdCyY7 } \\
\text { @ Sueleraci @nocompulsoryvac Actual, competent } \\
\text { science confirms vaccines DON'T cause ASD: } \\
\text { https://t.co/ENadr8G7MW }\end{array}$ \\
\hline Exortativo & $\begin{array}{c}5 \\
(1,6 \%)\end{array}$ & $\begin{array}{c}2 \\
(6,5 \%)\end{array}$ & $\begin{array}{l}\text { @lisascottbcd } \\
\text { Just in case you need a reminder, refresher, etc. } \\
\text { VACCINATE your children (that's me yelling) } \\
\text { https://t.co/OYvgw8GkeV }\end{array}$ \\
\hline
\end{tabular}


Ronaldo Ferreira de Araújo; Ariadne Chloe Mary Furnival

Comunicação científica e atenção online: em busca de colégios virtuais que sustentam métricas alternativas

\begin{tabular}{c|c|c|l}
\hline $\begin{array}{c}\text { Categoria } \\
\text { Analítica }\end{array}$ & Twitter & Facebook & \multicolumn{1}{c}{ Exemplos (tweets) } \\
\hline Problematizante & 1 & $\begin{array}{c}2 \\
(6,5 \%)\end{array}$ & $\begin{array}{l}\text { @NeilPCarlin @VaccineChoiceCA Table 2 raw data } \\
\sim 14 \% \text { greater \# of ASD Dx at 5 yrs with 1 dose MMR } \\
\text { vs unvax ?? https://t.co/yQqfKObW4e }\end{array}$ \\
\hline
\end{tabular}

Fonte: dados da pesquisa (2016).

A grande maioria dos tweets (186 ou 62\% da amostra de 300) se encontra na categoria Compartilhamento (repasse) e restringiu-se à replicação (retweets - RTs) do URL, título e/ou assunto do artigo. Este mesmo tipo de replicação da informação original foi representado por $45 \%$ das postagens no Facebook. Juntos, estas porcentagens representam várias possíveis dimensões de um comportamento informacional que, à primeira vista, poderia ser visto como "econômico" ou "apressado" no sentido que o tweeter ou postagem no Facebook simplesmente quer compartilhar a fonte original entre os participantes da sua comunidade. Este comportamento também pode ser interpretado como "neutro" pois ao meramente compartilhar a fonte original sem comentá-la, não é possível identificar "em qual lado" do argumento localiza-se o compartilhador (tweeter, poster) e pode ser que ela mesma nem sabe antes de ler o artigo (e possivelmente, um simples repasse do URL significa a não-leitura do artigo sendo referenciado).

A próxima categoria de tweets procura explicar um pouco mais os detalhes dos resultados do estudo, exemplos como "No higher risk with MMR even with siblings of autistic kids" (Sem maior risco com MMR mesmo com irmãos de crianças autistas) e "RT @DocBastard: .@bodhibrian The autism rate for unvaccinated kids (even high risk kids) is the same as vaccinated kids." ("A taxa de autismo para crianças não vacinadas (até mesmo crianças de alto risco) é a mesma que para crianças vacinadas") sendo representativos desta categoria. Os posts no Facebook que procuraram explicar o estudo eram predominantemente de associações de profissionais de saúde, alguns destes sumarizando e parafraseando os resultados do estudo.

Em mensagens de cunho conclusivas, chama a atenção o número substancial de tweets que realçam o tamanho grande da amostra (95.727 crianças) na pesquisa do artigo, tweets como "RT @sadhbhbyrne: Study of 100,000 children found no association between receipt of MMR vaccine and 
Autism Spectrum Disorders" e “@DocBastard: Study of over 95,000 children: 'No harmful association between MMR vaccine receipt and \#autism' e "@kath2cats: HUGE study. Autism rates unaffected by vaccine status" sendo bem representativos dessa categoria. A prioridade dada ao tamanho da amostra em tantos tweets conota a incontestável credibilidade científica atribuída ao estudo no artigo pelos tweeters.

A proporção (15\%) de tweets argumentativos que parecem estar respondendo a um outro interlocutor offscreen, como parte de uma discussão em andamento, também era esperada, devido à natureza da controvérsia em torno do tema da vacina, extremamente polarizada entre os anti-vacinadores e os pró-vacinadores, este último grupo aparentemente muito mais representado no Twitter. Exemplos de tweets captando estes fragmentos de conversas são "@qtbeauty Here's a paper not connected to the CDC. https://t.co/UTdWkq1QPI Read it."; "@thancockMD: "Current evidence disagrees with that assumption https://t.co/aYANu6so46"; "@ReversingASD @tepes74 feel free to argue amongst yourselves. You might also want to read this article. https://t.co/baeMOUZ2uO."

Os tweets que categorizamos como sendo do tipo exortativo, simplesmente citam a evidência apresentada no estudo como contundente em prol da advocacia para a vacinação: exortam aos pais a vacinar seus filhos como em "@lisascottbcd Just in case you need a reminder, refresher, etc. VACCINATE your children (that's me yelling) https://t.co/OYvgw8GkeV" e "@michaelbarakate. Get your kids vaccinated! JAMA this week- there is NO link between MMR and Autism!"

Por fim, chamou a atenção que na amostra randômica de tweets aqui examinados, nenhum questionou a credibilidade do estudo, o que não foi 0 caso para as postagens no Facebook, duas na amostra claramente questionando aspectos do estudo. Por exemplo, na página do Facebook 

métricas alternativas

chamada Vactruth.com, um grupo anti-vacina, lemos a seguinte postagem ${ }^{1}$ sobre o artigo em questão:

Per usual, a study has been released and widely published, stating that there is no correlation between autism and the MMR. Also per usual, it's not a real study. To date, there have been exactly zero double-blind studies which look at a vaccinated vs. an unvaccinated population. None. Zero. Zilch. The recent JAMA study is particularly deceiving. At face value, they state that the MMR does not cause autism. However, if you look at the data they're using to reach a conclusion, you'll see that it isn't actually a medical study, but an analysis of health insurance information...information that was intended for payment purposes, and not to form a conclusion regarding the safety or efficacy of vaccines.

Em outra página Facebook do grupo norteamericano, também antivacina, "Oregonians for Vaccine Truth and Healthcare Choice", uma postagem ${ }^{2}$ comenta o artigo publicada na JAMA, também comentando outros estudos semelhantes, da seguinte forma:

In this study [...] what was found repeatedly [...] was the use of flawed study designs, unreliable data, findings of obviously predetermined outcomes unsupported by the data, and major conflicts of interest in the studies funding sources. Keep in mind that no studies have ever been done in regard to the safety of the combined number of vaccines on the CDC vaccine schedule. No studies have been done on the safety of giving from 5 to up to 9 or more vaccines to a small child, in a single day and office visit. VACCINES AND AUTISM - WHAT DO EPIDEMIOLOGICAL STUDIES REALLY TELL US?

Em ambos os casos podemos perceber que os colégios virtuais identificados fomentam a polêmica em torno da vacina tríplice MMR (Measles, Mumps, Rubella - sarampo, caxumba, rubéola) - tópico do artigo cujos dados altmétricos são o foco - que encapsula a intersecção dos pontos da ciência (médica) com a incerteza científica e o risco no domínio público. Vale ressaltar que na mobilização em torno da "polêmica MMR", os relevantes atores já se

\footnotetext{
${ }^{1}$ Ver:

https://www.facebook.com/permalink.php?story_fbid $=10152938961912989 \&$ id $=287991907988$

${ }^{2}$ Ver: https://www.facebook.com/groups/OrSB132
} 
estenderam além dos grupos de pais, peritos médicos e profissionais de saúde para incluir uma pletora de jornalistas, políticos e advogados, e a polêmica já consolida movimentos em prol das liberdades cívicas e a escolha do paciente (p.ex. ao invés de receber uma vacina única, por que pais não podem reivindicar vacinas individuais?), da promoção da medicina alternativa e de anti-vacinação, e da proposição de conspirações entre grandes fármacos e médicos (LEACH, 2005).

Para a análise dos dados altmétricos sobre as mensagens que comentam o artigo-objeto do presente estudo, a polêmica em torno do MMR tem sido centrada numa tensão entre os tipos de estudo e concomitante metodologias de análise dos dados realizada.

\section{CONCLUSÃO}

A altmetria é um campo relativamente novo e ainda carece de mais estudos teóricos e empíricos para melhor a compreensão do que realmente suas métricas e indicadores representam para a comunicação científica. Sobretudo por ser calculada e verificada por diversas fontes com objetivos distintos e que podem representar diferentes significados em relação a circulação da informação científica. Independente de quais sejam as fontes dos dados altmétricos ao serem rastreadas as mesmas indicarão os atores, comunidades de atenção e colégios virtuais responsáveis pelas ações de informação de interação em torno dos itens que se analisa.

O emergente campo da altmetria propõe uma avaliação de impacto mais social e democrático da pesquisa científica dentro e fora da comunidade científica. Os colégios virtuais caracterizados e enquadrados na categoria de compartilhamento e repasse tem um público bem variado, já as categorias que expressam o debate sejam de cunho explicativo, conclusivo, argumentativo, exortativo ou problematizante indicam um público mais especializado e denotam que a circulação da informação científica sobre o artigo analisado se concentra na comunidade científica. 
O estudo demonstrou que prolifera a geração de dados altmétricos em torno de artigos científicos que veiculam informações sobre assuntos científicos percebidos como "controversos". A literatura sobre as controvérsias científicas relata como grupos dos lados "a favor" e "contra" da questão em pauta mobilizam e moldam a co-produção de posições científicas, sociais e políticas.

A rede do Twitter, por exemplo, se configura como fórum ideal para a consolidação de tais posições. No entanto, o estudo demonstrou menos uma rede representando uma miríade de posicionamentos, e mais uma rede que replica "fertilmente", uma ou outra posição: os dados altmétricos revelam muito menos variedade de posições - captadas nas "menções" do artigo sob análise, que se esperava.

\section{REFERÊNCIAS}

ALLEN, R. What happens online in 60 seconds? Smartinsights. Disponível em: http://www.smartinsights.com/internet-marketing-statistics/happens-online-60seconds/; Acesso em: 19 ago 2016.

ALTMETRIC. How is the Altmetric score calculated? 2014. Disponível em:<http://support.altmetric.com/knowledgebase/articles/83337-how-is-the-altmetricscore-calculated- > Acesso em 07 jan., 2015.

ALPERIN, J. P. Ask not what altmetrics can do for you, but what altmetrics can do for developing countries. Bulletin of the American Society for Information Science and Technology, v. 39, n. 4, p. 18-21, 15 abr 2013.

ARAÚJO, V. M. R. H.; FREIRE, I. M.. A rede Internet como canal de comunicação, na perspectiva da Ciência da Informação. Transinformação, Campinas, v.8, n.2, maiago, p.45-55, 1996.

ARAÚJO, R. F.; MURAKAMI, T. R. M. A.. Atenção online de artigos de ciência da informação: análise a partir de dados altmétricos do Facebook In: ENCONTRO BRASILEIRO DE BIBLIOMETRIA E CIENTOMETRIA, 5., 2016, São Paulo. Anais... São Paulo: USP, p.1-8, 2016.

ARAUJO, R. F.. Marketing científico digital e métricas alternativas para periódicos: da visibilidade ao engajamento. Perspectivas em Ciência da Informação, v. 20, p. 67-84, 2015.

BENGSCH, D.. 8 out of 8 million: Today we celebrate eight million researchers and scientists on ResearchGate. ResearchGate Blog, 8 out., 2015. Disponível em: < https://www.researchgate.net/blog/post/8-out-of-8-million > Acesso em 21 ago., 2016. 
CASTRO, Regina C. Figueiredo. Impacto da Internet no fluxo da comunicação científica em saúde. Rev. Saúde Pública, São Paulo, v. 40, n. spe., ago. 2006.

CAVE, R. Overview of the Altmetrics Landscape. Proceedings of the

Charleston Library Conference, Purdue University Press, Indiana, 2012.

Disponível em:

http://docs.lib.purdue.edu/cgi/viewcontent.cgi?article=1381\&context=charleston; Acesso em 17 ago 2016.

FAUSTO, S. et al. Research Blogging: Indexing and Registering the Change in Science 2.0. Plos One, v. 7, p.e50109, 2012.

GARVEY, W. D.; GRIFFITH, B. C.. Scientific communication in social system. Science, 157, p.1011-1016, set., 1967.

GURGEL, N. M. C. ; MATOS, D. M. Diagnóstico das pesquisas virtuais da comunidade científica do Labomar. In: SEMINÁRIO NACIONAL DE BIBLIOTECAS UNIVERSITÁRIAS, 14., 2006, Bahia. Anais... Bahia: UFBA, 2006.

HAUSTEIN, S.; BOWMAN, T. D; COSTAS, R. "Communities of attention" around scientific publications: who is tweeting about scientific papers? Work in progress. Presented at the Social Media \& Society 2015 International Conference, Toronto, Canada, p.1-20, 2015.

HORNBY, A.; LESLIE, B.. Digital scholarship and scholarly communication. University of Washington Libraries. 2013.

LE COADIC, Y.-F. A Ciência da Informação. Brasília: Briquet de Lemos, 1996. $119 \mathrm{p}$.

MEADOWS, Arthur. A comunicação científica. Brasília: Briquet de Lemos, 1999

MOREIRA, Valter. Os colégios virtuais e a nova configuração da comunicação científica. Ciência da Informação. Brasília, v. 34, n. 1, p.57-63, jan./abr. 2005.

MUELLER, S. A ciência, o sistema de comunicação científica e a literatura científica. In: CAMPELLO, B.S.; CENDÓN, B.V.; KREMER, J.M. (orgs). Fontes de informação para pesquisadores e profissionais. Belo Horizonte: Ed. UFMG, 2000.

NELHANS, G.; LORENTZEN, D.G. Twitter conversation patterns related to research papers. Information Research, v.21, n.2, paper SM2. Disponível em: http://InformationR.net/ir/21-2/SM2.html; acesso em 14 set. 2016. 
NEUBERT, P. S.; RODRIGUES, R. S.. Pesquisa bibliográfica e web 2.0: percepção de estudantes de pós-graduação em ciência da informação. Inf. \& Soc.:Est., João Pessoa, v.22, n.3, p.143-154, 2012.

OLIVEIRA, E. B. P. M. de; NORONHA, D. P. A comunicação científica e o meio digital. Inf. \& Soc.:Est., v. 15, n. 1, 2005.

PRIEM, Jason; PIWOWAR, Heather A.; HEMMINGER, Bradley M. Altmetrics in the wild: using social media to explore scholarly impact. arXiv, p. 1-17, 20 mar. 2012. Disponível em: < http://arxiv.org/html/1203.4745v1 >. Acesso em 24 mar., 2013.

PRIEM, J. et al. Altmetrics: a manifesto. 2010. Disponível em: <http://altmetrics.org/manifesto>. Acesso em: 02 abr. 2014.

RUMSEY, A.. New-Model Scholarly Communication: road map for change. Scholarly Communication Institute 9. University of Virginia Library. 2011.

SILVA, E. L.; MENEZES, E. M.; BISSANI, M.. A internet como canal de comunicação científica. Inf. \& Soc.:Est., João Pessoa, v.12, n.1, p.1-17, 2002.

TANANBAUM, G.. Article-Level Metrics: a sparc primer. Sparcopen. Abr, 2013. Disponível em: < http://www.sparc.arl.org/sites/default/files/sparc-alm-primer.pdf $>$ Acesso em 13 ago., 2016.

WHITE, M. D.; MARSH, E. E. Content analysis: a flexible methodology. Library Trends, v.55, n.1, p.22-45, 2006.

Title

Scientific communication and online attention: in search of virtual colleges underpinning altmetrics data

\begin{abstract}
Introduction: Informational exchanges around scientific communication activity have become increasingly dynamic in cyberspace, changing the practice of scientists and expanding the reach of research results that attain an increasingly significant online attention among different groups.

Aim: To analyze whether altmetric data regarding online attention point to the existence of "virtual colleges" - composed of people from inside and outside the scientific community - around the scientific topic being discussed and shared in social media.

Methodology: An exploratory pilot study, with analysis focused on the impact of the open access article with the highest altmetric indicator for the year 2015, as disclosed by Altmetric.com in its ranking "Top 100". The qualification of online attention is described by the most expressive media (Twitter and Facebook) in a random sample ( $\mathrm{n}$ $=10 \%$ ) on which content analysis was carried out and categorization of the types of interaction and characterization of social groups that interacted around the article.
\end{abstract}


Results: The most common form of dissemination observed was retweeting on Twitter $(62 \%)$ and sharing on Facebook (45\%), followed by "conclusive" (16\% and $25 \%)$ and "argumentative" (15\% and 9.6\%) categories. The profile of the users responsible for the circulation of and discussion about the article indicates the prevalence of members from the general public.

Conclusion: Altmetrics permit the tracking of the potentially more social and democratic impact assessment of scientific research, which becomes particularly evident for the case of articles featuring scientific research deemed to epitomise scientific controversies, the dimensions of which are played out in these "virtual colleges".

Keywords: Scientific communication. Altmetrics. Online attention

\section{Titulo}

Comunicación científica y atención en línea: en búsqueda de colegios virtuales que apoyan las altmétricas

\section{Resumen}

Introducción: Los intercambios de información alrededor de la actividad de comunicación científica se han llegado a ser más dinámicos en el ciberespacio, modificando la práctica de los científicos y ampliando el alcance de los resultados de la investigación que alcanzan una atención en línea cada vez más importante entre los diferentes grupos.

Objetivo: Analizar si los datos altmétricos de la atención en línea indican la existencia de "colégios virtuales" - compuestos por personas de dentro y fuera de la comunidad científica - sobre el tema científico en discusión y compartido en las redes sociales.

Metodología: Un estudio piloto, caracterizado como exploratoria, con el análisis se centrando en el impacto del artículo de acceso abierto con el indicador altmétrico más alto para el año 2015, como publicado por Altmetric.com en su ranking "Top 100". La calificación de la atención en línea es descrita por los medios más expresivos (Twitter y Facebook) en una muestra aleatoria $(n=10 \%)$ para la que se condujo el análisis de contenido y clasificación de los tipos de formas de interacción y caracterización de los grupos sociales que interactúan.

Resultado: La forma más común de propagación observada fue la reproducción para el Twitter (62\%) y el compartir para el Facebook (45\%), seguido por categorías "concluyentes" (16\% y $25 \%$ ) y "argumentativo" (15\% y $9,6 \%)$. El perfil de los usuarios responsables de la circulación del artículo indica que son predominantemente los miembros del público en general.

Conclusión: Aunque las Altmetrics propongan una evaluación del impacto social y democrático de la investigación científica, los colégios virtuales destacadas indican un público especializado e indican que el debate y la difusión de información científica sobre el artículo analizado se limita a la comunidad científica.

Palabras clave: comunicación científica. Altmetrics. atención en línea

Enviado em: 17.07.2016

Aceito em: 20.11.2016. 